University of Nebraska - Lincoln

DigitalCommons@University of Nebraska - Lincoln

2010

\title{
Age effect on the Gaze Stabilization test
}

Julie A. Honaker

University of Nebraska at Lincoln, jhonaker2@unl.edu

Follow this and additional works at: https://digitalcommons.unl.edu/specedfacpub

Part of the Special Education and Teaching Commons, and the Speech Pathology and Audiology Commons

Honaker, Julie A., "Age effect on the Gaze Stabilization test" (2010). Special Education and Communication Disorders Faculty Publications. 60.

https://digitalcommons.unl.edu/specedfacpub/60

This Article is brought to you for free and open access by the Department of Special Education and Communication Disorders at DigitalCommons@University of Nebraska - Lincoln. It has been accepted for inclusion in Special Education and Communication Disorders Faculty Publications by an authorized administrator of DigitalCommons@University of Nebraska - Lincoln. 


\title{
Age effect on the Gaze Stabilization test ${ }^{1}$
}

\author{
Julie A. Honaker ${ }^{\mathrm{a}, *}$ and Neil T. Shepard ${ }^{\mathrm{b}}$ \\ ${ }^{a}$ Department of Special Education and Communication Disorders, University of Nebraska, Lincoln, NE, USA \\ ${ }^{\mathrm{b}}$ Balance/Vestibular Program, Division of Audiology, Mayo Clinic, Rochester, MN, USA
}

Received 23 September 2009

Accepted 23 March 2010

\begin{abstract}
Impairments of the vestibular-ocular reflex (VOR) lead to a decline in visual acuity during head movements. Dynamic visual acuity (DVA) testing is a sensitive assessment tool for detecting VOR impairments. DVA evaluates accuracy of visual acuity during fixed velocity head movements. In contrast, the Gaze Stabilization test (GST) is a new functional evaluation of the VOR that identifies a person's maximum head velocity (in degr ees per second) a person can maintain with stable vision of a target (i.e. optotype). The objective of this study was to evaluate the effect of age on the GST in participants without vestibular disease. The study was conducted in a vestibular and balance laboratory at a tertiary medical center. A total of 87 healthy adult volunteers were included in this study. The main outcome measure was the association between age and both GST maximum head velocity in the yaw (right/left) plane and velocity symmetry. A significant negative correlation was found between age and maximum head velocity $(r=-0.469, p<0.001)$. Our results suggest that age should be considered when interpreting GST results in the yaw plane, however standardization of testing methods should be established as variation in results has been reported in the literature.
\end{abstract}

Keywords: Visual acuity, vestibular function test, vestibular-ocular reflex, gaze stabilization, dynamic visual acuity

\section{Introduction}

In order to maintain stable vision during head movements our vestibular-ocular reflex (VOR) and other eye mechanisms that produce compensatory eye translations keep the image of interest on the fovea. VOR gain decreases with age and results in poorer visual acuity during head movements due to significant retinal slip [2]. Unlike other physiologic tests of the VOR (e.g. caloric subtest of electronystagmography and rotational chair), functional measures of gaze stability, such as Dynamic Visual Acuity testing (DVA) have been used routinely as part of assessment for individuals with vestibular hypofunction. DVA is a measure of visual acuity that investigates the smallest target (opto-

* Corresponding author: Julie Honaker, PhD, 272 Barkley Memorial Center, University of Nebraska-Lincoln, Lincoln, NE 685830738, USA. Tel.: +1 402472 5493; Fax: +1 402472 7697; E-mail: Jhonaker2@unl.edu.

${ }^{1}$ A portion of this paper was presented at the American Academy of Audiology's annual conference on April 1-4, 2009 in Dalla s, TX, USA. type) an individual can observe accurately during horizontal head movements. While DVA has shown good sensitivity and specificity for individuals with vestibular disorders [5] the use of a progressively decreasing optotype may affect the results for individuals with eye disorders affecting visual acuity [4]. The Gaze Stabilization test (GST) was introduced as new functional evaluation of a person's ability to use the VOR for appropriate visual target capture during head movements through FDA approved computer software. GST identifies maximum head velocity (in degrees per second) while maintaining clear visual fixation [4]. An optotype "E" presented at a fixed target size is randomly presented to the patient for a brief period during the active headshake movement to determine the maximum speed that the subject can observe the target in focus. Goebel, Tungsiripat, Sinks and Carmody [4] established an estimate of the sensitivity of the GST for identifying unilateral vestibular deficits, using normal subjects compared to those with vestibular dysfunction at $64 \%$ and the specificity at $93 \%$. The authors concluded that the peak head velocity (in the yaw plane) recorded from 
GST provided a valuable marker for assessing treatment outcome and evaluating the degree of functional activity as compared to Dynamic Visual Acuity testing. However, the effects of age on GST results were not established. Recently, Pritcher, Whitney, Marchetti, and Furman [11] demonstrated no significant difference in maximum GST velocity values during yaw or pitch plane head movements between 20 young control subjects ( $20-40 \mathrm{yrs})$ and 20 older controls (60-80 yrs). The current project was proposed to broaden the age range of participants without vestibular disease and determine if there is an age effect on GST as normative data is needed for properly examining the GST results with vestibular disorders.

\section{Materials and methods}

\subsection{Subjects}

Eight-nine healthy volunteers were recruited from community sources in Rochester, Minnesota and consented to participate in the institutional review board approved study. Volunteers were excluded if they reported history of any form of dizziness (including complaints of lightheadedness, vertigo, unsteadiness) lasting longer than 1 hour or recurring for greater than 1 day; 2) history of any disorder interfering with neck range of motion; 3) history of known neurological diagnosis or musculoskeletal injuries affecting the subject's ability to generate active cervical rotation; 4) his tory of eye disorders that might affect visual acuity (i.e. cataracts, macular degeneration, diabetic retinopathy). Two subjects were excluded from participation in the study based on history of eye disorders affecting visual acuity. The remaining eighty-seven participants (26 males and 61 females) were placed into an appropriate age group as follows: Group 1 consisted of fifteen subjects ranging in age from 20-29; Group 2 included fourteen subjects ranging in age from 30-39; Group 3 was composed of fifteen subjects ranging in age from 40-49; Group 4 consisted of fifteen subjects ranging in age from 50-59; Group 5 consisted of fifteen subjects ranging in age from 60-69; and Group 6 included thirteen subjects ranging in age from 70-79 years. The investigator performed direct case history to review health status to determine if subjects meet the study requirements. Corrective lenses history was collected on all participants and revealed 11,13 and 33 with bifocals, contacts and regular lenses, respectively. One subject wore trifocals and 1 wore progressive lenses during testing.

\subsection{Test protocol}

Visual acuity with and without head movements was measured using Neurocom ${ }^{\circledR}$ International software, version 8.3.0. Each subject sat in a well-lit room in front of a computer screen ten feet from the plane of their eyes. Static visual acuity (SVA) was measured by displaying sequences of an optotype " $E$ " in random orientations (i.e. up, down, right and left). The smallest "E" that could be identified at least 3 of 5 successive presentations was given in $\log$ MAR units ( $\log$ of the Minimum Angle of Resolution). Perception Time Test (PTT) was initiated after SVA baseline scores were established. During the PTT, subjects were asked to again identify the orientation of the optotype "E" at a size of $0.2 \log$ MAR above the subject's SVA that flashed on the computer screen. The PTT determined the minimum target presentation time in msec. via a protocol where the optotype at $0.2 \log$ MAR greater than SVA was able to be identified accurately 3 out of 5 times. This presentation time was used in GST testing.

\subsubsection{Gaze Stabilization test protocol}

A head-mounted rate sensor (InertiaCube2 Precision Motion Tracker) was initialized via an internal process that determined its position in three dimensional space relative to gravity [9]. This was performed prior to placing the device on the subject's head. All subjects were instructed to move their head in the yaw plane at $20 \mathrm{deg}$ excursions to the right and left and slowly increase head movements until they reached a preset starting velocity of 50 degrees per second. At this point, an optotype "E", set at $0.2 \log M a r$ above the subject's SVA score and based on individual PTT score, appeared. Neurocom ${ }^{\circledR}$ International software determined threshold response ( $60 \%$ correct $3 / 5)$ based on the psychophysical adaptive process, PEST (Parameter Estimation by Sequential Testing) algorithm [6].

\subsection{Statistical analysis}

The mean and standard deviation of the GST results in the healthy subjects were calculated. Linear regression and correlation coefficients were used to assess the association between age and both GST maximum velocity in yaw (right/left) and velocity symmetry. In addition, comparison was made between healthy young (20-39 yrs) to healthy old (60-79 yrs) using two sample $t$ tests for each of the measurements (maximum velocity and velocity symmetry). Paired $t$-test was used to compare overall GST maximum velocity values within 
subjects. All statistical calculations were performed using PASW (Version 18, SPSS Inc., Chicago, IL). Pvalues $<0.05$ were considered statistically significant. Standard parametric statistics were used as our sample population was found to be normally distributed via use of chi square analysis comparing a normal distribution to the histogram of mean velocity values.

\section{Results}

The final sample consisted of 87 participants, Group $1=15$ subjects (20-29 years), mean age $25.2 \pm 2.59$ years; Group $2=14$ subjects ( $30-39$ years), mean age $34.21 \pm 2.91$ years; Group $3=15$ subjects $(40-49$ years), mean age $44.6 \pm 2.80$ years; Group $4=15$ subjects ( $50-59$ years), mean age $54.27 \pm 3.03$ years; Group $5=15$ subjects ( $60-69$ years), mean age 63.87 \pm 2.97 years; Group $6=13$ subjects ( $70-79$ years), mean age $73.85 \pm 2.91$ years. Table 1 summarizes the head velocity findings for the GST and perception time scores. When we evaluated the relationship between age and maximum GST velocity values we found a significant negative correlation with age (see Fig. 1). There was not a significant correlation between age and velocity symmetry percentage values $(r=-0.01$, $p=0.94$ ). There was also no significant difference in overall GST maximum velocity values within subjects $(p=0.232)$. The mean perception time score was $41.56 \mathrm{msec}$. There was a significant difference in perception time scores across groups $(p=0.026)$. Of the 59 subjects who wore corrective lenses during testing, we found no significant difference in performance as compared to the 28 subjects without corrective lenses $(p=0.317)$

Groups 1 and 2 were combined to create a young subject group (ages 20-39 yrs) and groups 5 and 6 were combined to create an older subject group (60-79 yrs) to specifically examine performance differences between these two age extremes. Again, there was a significant age effect, where older subjects (60-79 yrs) demonstrated a significantly slower GST maximum velocities (mean $114.70 \pm 28.95$ vs. $154.72 \pm 34.09, p<$ 0.001 ) than younger (20-39 yrs) subjects (Fig. 2).

\section{Discussion}

Visual acuity has been recognized to worsen with age $[3,10]$. Previous work has identified an age effect on dynamic visual acuity testing $[1,7,12]$. Op- tical changes such as lens thickening and changes in pupillary constrictions may account for the age-related changes [7].

The results of this study support previous research concerning overall age affect on functional testing (active gaze stability) of the vestibular-ocular reflex [7]. In particular, we found a significant difference in GST average velocity across age groups spanning from 20 79 years and when comparing age group extremes (i.e. young (20-39 yrs) and older (60-79 yrs). However, an unexpected finding in this study was the maximum head velocities during the GST for Group 4 (50-59 years) exceeded those for Group 3 (40-49 years) as reported in Table 1. While significant changes in GST scores were appreciated in later decades of life, it is possible that more factors may play a role in GST velocity score variability for middle decades of life such as the role of visual correction, additional health affects, and overall activity levels. The small sample size collected for each group may be affecting the results as well.

Our significant age effect differs from that recently reported by Pritcher et al. [11]. They report that there were no significant difference in GST velocity scores between 20 old (60-80 years of age) and 20 young ( 20 40 years) healthy subjects. We speculate that one possible reason the age effects reported herein differed from those of Pritcher et al. is due to differences in optotype presentation time (i.e. perception time test results). The InVision software version used in the study by Pritcher et al was not reported, so it is difficult to determine whether or not target presentation time was at a fixed rate (as proposed in older InVision software versions) or if presentation time was variable based on perception time test scores. One can assume that the perception time was variable as the authors stated that the optotype presentation rate was $75 \mathrm{msec}$ or less; whereas Goebel et al. [4] used a fixed presentation time of $75 \mathrm{~ms}$ for all participants. Based on our perception time test scores, our optotype presentation rates were $70 \mathrm{msec}$ or less, with a significant difference in PTT among age groups. It is possible that the individual subjects in this study had a greater optotype time (i.e. longer presentation in msec) than those reported by Pritcher et al. This increased optotype presentation time may have allowed our subject pool a longer opportunity to view the optotype ultimately resulting in better performance (i.e. increased head speed) across all age groups. While this may have contributed to the better performance, it should be noted that there was a significant increase in perception time with age. Based on the inVision software protocol, once the subject's perception time score 
Table 1

Gaze Stabilization maximum head velocity results and perception time scores (mean \pm standard deviation $(\mathrm{SD})$

\begin{tabular}{ccc}
\hline Subject group & $\begin{array}{c}\text { Maximum velocity degree/s } \\
\text { (mean } \pm \mathrm{SD} \text { ) }\end{array}$ & $\begin{array}{c}\text { Perception time score } \\
\text { (mean } \pm \mathrm{SD} \text { ) }\end{array}$ \\
\hline Group 1 (20-29 yrs) $n=15$ & $158.43 \pm 35.88$ & $22.67 \pm 7.04$ \\
Group 2 (30-39 yrs) $n=14$ & $150.75 \pm 32.92$ & $22.86 \pm 7.26$ \\
Group 3 (40-49 yrs) $n=15$ & $132.67 \pm 39.02$ & $32 \pm 14.74$ \\
Group 4 (50-59 yrs) $n=15$ & $147.40 \pm 28.54$ & $28 \pm 10.14$ \\
Group 5 (60-69 yrs) $n=15$ & $123.23 \pm 30.14$ & $29.33 \pm 14.86$ \\
Group 6 (70-79 yrs) $n=13$ & $104.84 \pm 25.11$ & $36.15 \pm 16.09$ \\
\hline
\end{tabular}

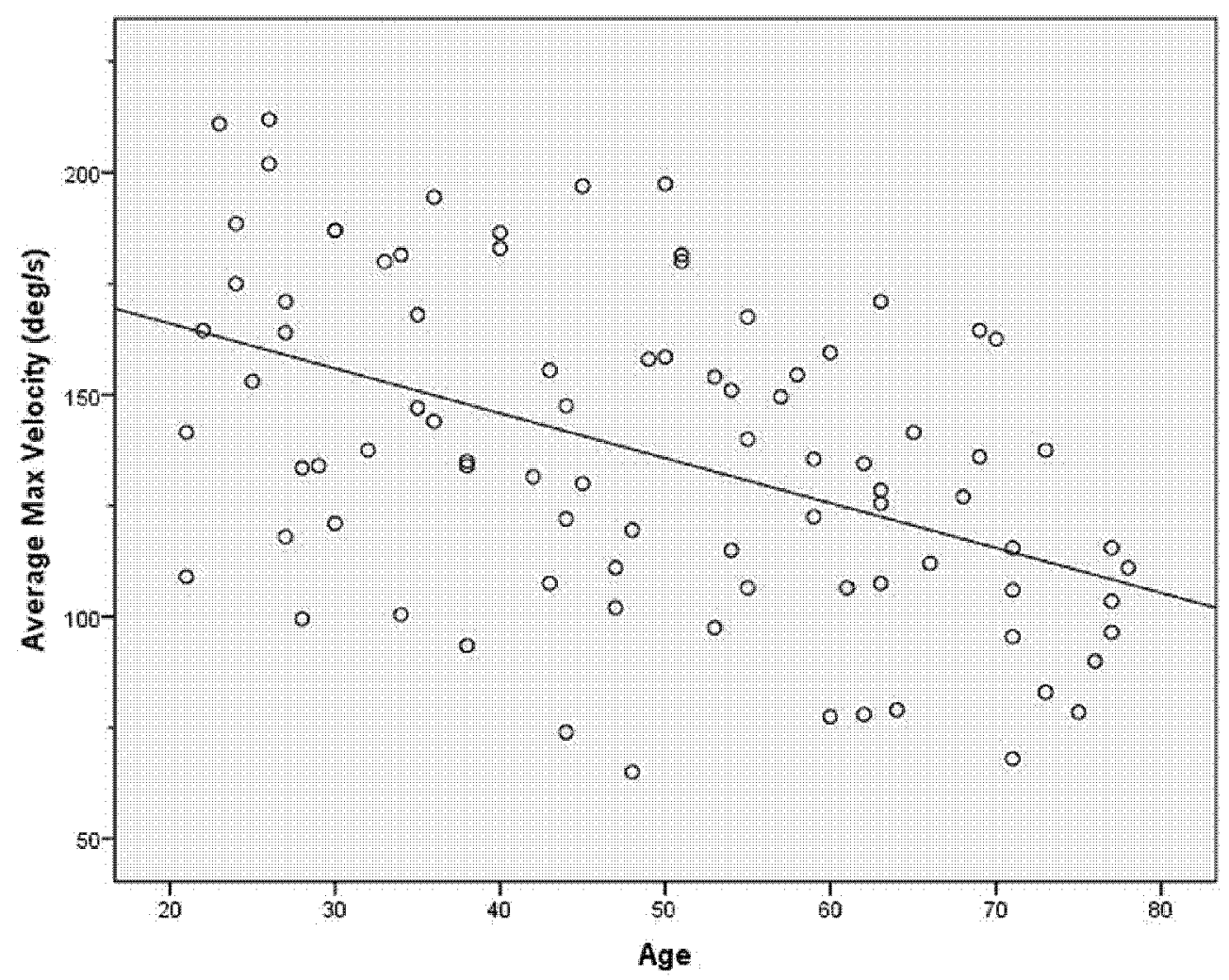

Fig. 1. Relationship between age and average maximum head velocity $(\mathrm{deg} / \mathrm{s})$. There was a significant negative correlation between age and average velocity $(r=-0.469, p<0.001)$.

was established the target was presented at the threshold of the measured perception. One may question presenting a target at threshold rather than displaying the target at a fixed time (in msec) above threshold as that might have caused some of the elderly individuals more trouble with target acquisition and hence a lower peak velocity during the GST. To address this concern, we recommend future work to evaluate the effects of the GST performance when using perception time threshold verses a fixed increased in time above threshold in order to properly standardize the GST.

Our yaw plane velocity scores are similar to those reported by Goebel et al. However, it should be noted that in our experiment we used a logMAR of 0.2 above SVA at a distance of 10 feet; whereas Goebel et al. used a $\log$ MAR of 0.3 above the SVA at a distance of 4.9'. Pritcher et al. also reported using a $\log$ MAR of 0.2 above SVA and speculated the difference between their work and that of Goebel's for maximum velocity related to the lower $\log$ MAR (i.e. 0.3 vs. 0.2 above SVA) causing a smaller optotype size and may have caused individual subjects to decrease their head speed in order to view the target. While we are in agreement with this, it should be noted that we used the same $0.2 \log$ MAR as Pritcher et al. but changed the viewing distance from $4.9^{\prime}$ to $10^{\prime}$. One may argue that the 


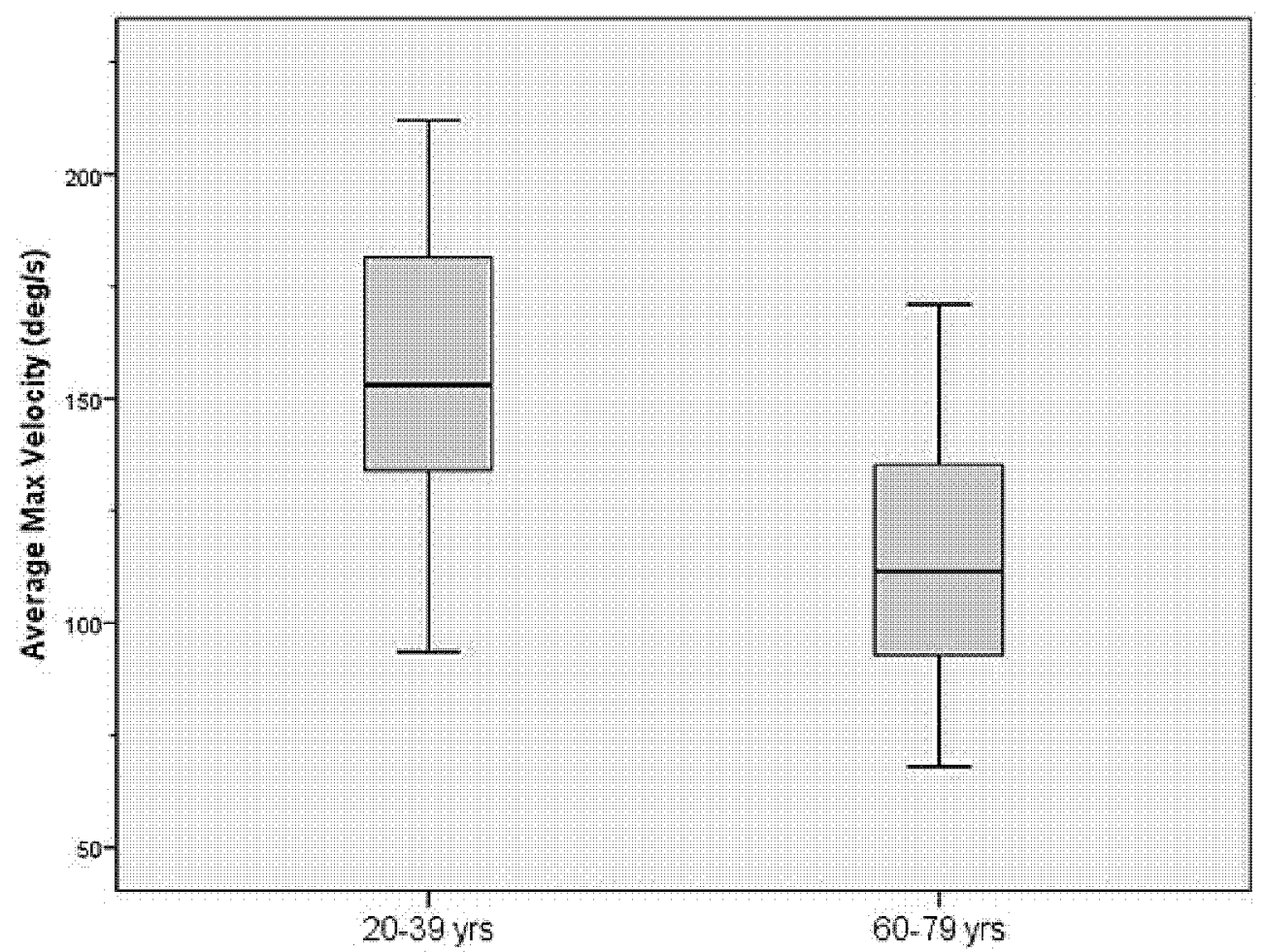

Fig. 2. Box plots of the medium (center line within the boxes) and interquartile range (upper and lower boarders of the boxes) of GST velocities ( $\mathrm{deg} / \mathrm{s})$ for young subjects ( $20-39 \mathrm{yrs}$ ) and older subjects $(60-79 \mathrm{yrs}), p<0.001$. The complete range of data in degs/s (minimum to maximum) for the younger subjects was 93.50 to $212 \mathrm{deg} / \mathrm{s}$; the data range for older subjects was 68 to $171 \mathrm{deg} / \mathrm{s}$.

decrease in target distance as reported by Pritcher et al. may have increased VOR gain; specifically, as the target comes closer for a given head speed the eye has to move further to maintain the target and therefore increasing the likelihood of retinal slip error. As pertained to the GST, this should result in reducing the head speed that individual subjects may be able to maintain to capture the target. However, the difference in vergence angle for targets at $4.9^{\prime}$ vs $10^{\prime}$ would only be $\sim 1$ degree. It is very unlikely that this would cause change in GST velocities. Migliaccio et al. [8] have shown that very near targets $(15 \mathrm{~cm})$ are needed for strong vergence effects on increasing VOR gain.

It should also be noted that with the 'E' optotype, the nature of blurring of the visual target is different with horizontal head movement when the ' $E$ ' is pointed to the sides verses when it is pointed up or down. The authors did not observe any difference during these conditions; however, based on the results obtained with the inVision software there is no scientific way for us to address that potential concern as the data was not sorted in respect to optotype orientation. From a qualitative standpoint, the authors did not receive any unsolicited comments to that effect from the subjects.
The use of corrective lenses has been documented in the literature $[4,11]$ but the effect of visual correction during GST testing has not been reported. While we had 59 out of 87 individuals wearing corrective lenses during GST evaluation, 13 of whom wore bifocals, trifocals or progressive lenses, we did not find a significant difference in performance between those without corrective lenses verses those with corrective lenses. It should be noted that the effect of corrective lenses on the GST was not a primary aim of this study and measurements of correction strength were not recorded for analysis. Future research is necessary to quantify the effects of corrective lenses on GST performance.

\section{Conclusion}

Gaze stabilization testing has been determined to be a functional evaluation of vestibular-ocular reflex. Our findings suggest that age should be taken into consideration when interpreting GST results in the yaw plane assuming protocol parameters used herein. Future research is needed to determine normative data for age- 
related effects in the pitch plane. Also, standardization of testing method needs to be established as there is a concern for variation in results among laboratories. Our testing parameters described should not be considered optimal as the parameters chosen were ones of convenience given the inVision software used, resolution of the NeuroCom computer screen, lighting conditions of the test facility, and selected distance from the target. Varying parameters resulting in larger, brighter targets in more dimly lit rooms will result in higher peak velocities. This is why standardization of this test is critical for comparison of results across facilities. We do not have data from this study to analyze the varying effects of the parameters used and how much each of the variables changed with respect to age. With data of this type, recommendations could be made for optimal parameters. We are however in the process of evaluating these parameters in a single study to determine what combination of parameters seems to provide optimal results for standardization of GST testing across facilities.

\section{References}

[1] A. Burg, Visual acuity as measured by dynamic and static tests: a comprehensive evaluation, J Appl Psychol 50(6) (1966), $460-466$.
[2] B.T. Crane and J.L. Demer, Human gaze stabilization during natural activities: translation, rotation, magnification, and target distance effects, J Neurophysiol 78 (1997), 2129-2144.

[3] D.B. Elliott, K.C. Yang and D. Whitaker, Visual acuity changes throughout adulthood in normal, healthy eyes: seeing beyond 6/6, Optom Vis Sci 72(3) (1995), 186-191.

[4] J.A. Goebel, N. Tungsiripat, B. Sinks and J. Carmody, Gaze stabilization test: a new clinical test of unilateral vestibular dysfunction, Otol Neurotol 28 (2007), 68-73.

[5] S.J. Herdman, R.J. Tusa and P. Blatt, Computerized dynamic visual acuity test in the assessment of vestibular deficits, $\mathrm{Am}$ J Otolaryngol 19 (1998), 790-796.

[6] M.R. Leek, Adaptive procedures in psychophysical research, Percept Psychophys 63(8) (2001), 1279-1292.

[7] G.M. Long and R.F. Crambert, The nature and basis of agerelated changes in dynamic visual acuity, Psychol Aging $\mathbf{5}(1)$ (1990), 138-143.

[8] A.A. Migliaccio, L.B. Minor and J.P. Carey, Vergencemediated modulation of the human angular vestibule-ocular reflex is unaffected by canal plugging, Exp Brain Res 186 (2008), 581-587.

[9] NeuroCom International, Inc. InVision Options GST, in: Instructions for Use: Equites ${ }^{\circledR}$ System Operator's Manual, Neuro Com International, Inc.: Clackamas, OR.

[10] D.G. Pitts, Visual acuity as a function of age, J Am Optom Assoc 53 (1982), 117-124.

[11] M.R. Pritcher, S.L. Whitney, G.F. Marchetti and J.M. Furman, The influence of age and vestibular disorders on gaze stabilization: a pilot study, Otol Neurotol 29 (2008), 982-988.

[12] M.C. Schubert, S.J. Herdman and R.J. Tusa, Vertical dynamic visual acuity in normal subjects and patients with vestibular hypofunction. Otol Neurotol 23(3) (2002), 372-377. 